\title{
Review of acupuncture and female urology
}

\author{
Kenneth $\mathrm{Ng}^{1}$, Ashanda Esdaille ${ }^{1}$, Mitchell $\mathrm{Ng}^{2}$ and Wellman W Cheung ${ }^{1}$ \\ ${ }^{1}$ Department of Urology, State University of New York Downstate Medical School, USA \\ ${ }^{2}$ Case Western Reserve University School of Medicine, USA
}

\section{Brief history of Chinese acupuncture}

Acupuncture, the insertion of thin needles at specific points in the body, is a form of alternative medicine with roots in the Han Dynasty of ancient China. The first written record is found in "The Yellow Emperor's Classic of Internal Medicine" published around 100 BC [1]. Documents, dating even earlier to 4,000 years ago, present the ancient works of Dr. Fu Xi which specify techniques with therapeutic stone needles, as well as Huang Di's invention of acupuncture and moxibustion [2]. Acupuncture has its philosophical roots in traditional Chinese medicine of the Shang dynasty, which dates back to 3000 years ago, and in the philosophy of the Ying Yang and the five elements. The Zhen Jiu Jia Yi Jing, the oldest acupuncture book in existence, emerged in the $1^{\text {st }}$ century AD [3]. By the Ming dynasty, acupuncture was formally recognized by the Imperial Medical Institute in China and incorporated into its teachings.

Acupuncture found its way to the Westin the $17^{\text {th }}$ century. At the time, missionaries, traders and physicians working in China were the first to be exposed to the practice. The surgeon-general of the Dutch East India Company published De Acupunctura in 1683, describing acupuncture to Western audiences based on interactions with Eastern acupuncturists. Jesuit missionaries from France also imported the practice to their clinics. In fact, the word "acupuncture" was introduced by the French Jesuits from the Latin words "acus" and "punctura," which meant needle and puncture, respectively [4]. Acupuncture continued to spread across the West throughout the Victoria Era and $19^{\text {th }}$ century, but nonetheless found limited interest and practice.

In the modern era, acupuncture found its way to the U.S. after Richard Nixon's historic visit to China. In 1971, James Reston, vice president of the New York Times, described being treated for postappendectomy abdominal distention with acupuncture [5]. The first FDA-approved acupuncture center was founded in 1972, and acupuncture was recognized by the IRS in 1973 as a deductible medical expense [6]. Nevertheless, research studies in the West were sporadic until 1976 when the FDA first classified acupuncture needles as investigational devices (Class III). In 1996, the FDA further reclassified acupuncture needles to Class II, a category that allows for general use by licensed practitioners [7]. In 2003, there were 20,750 active acupuncturist licenses in 39 states, with California accounting for $38 \%$ [7]. In 2007, there are more than 55 accredited acupuncture programs or schools. Forty eight states are now regulating statues [8]. More medical physicians, including neurologists, anesthesiologists, and specialists in physical medicine, are becoming trained in acupuncture.

\section{Principles of acupuncture}

According to the World Health Organization (WHO), a broad definition of acupuncture is the stimulation of certain points on the body (acupuncture points) using needling, moxibustion, electricity, laser, or acupressure for therapeutic purposes [9]. There are several types of acupuncture: Traditional Chinese Medicine acupuncture, French energetic acupuncture, Korean hand acupuncture, auricular acupuncture, myofascial acupuncture and Japanese acupuncture. Although each has its unique characteristics and differing locations of acupuncture points, all are based on one of the following types of stimulation: manual, electro-acupuncture, heat, laser and moxa [4]. Acupuncture has historically been used for varied therapeutic purposes, but most often utilized for pain management and analgesic purposes, primarily musculoskeletal and neuropathic pain. Currently, the American Society of Anesthesiologists' Task Force on Chronic Pain management states that acupuncture may only be used for pain management in conjunction with conventional anesthetic therapy [10].

In Eastern philosophy, acupuncture is based on the principle of promoting balance of the Ying-Yang through realigning Qi (chee) or energy flow in the body. Qi is believed to from "inside" organs to "outside" tissues like skin and bone. The flow occurs through meridians where the acupuncture needles are inserted [11]. For many years, the consensus view within Western medicine was that acupuncture was based on shabby pseudoscience with no logical or legitimate mechanism of actions [12]. Though, the emerging and more modern Western medical view of acupuncture is that like other forms of traditional and alternative medicine; it may indeed have therapeutic effects not previously recognized. Acupuncture has been theorized to cause release of endorphins which provide analgesic effects [13]. It has been shown to cause release of adenosine which can mediate pain control [14]. Through stimulation of the vagus nerve, acupuncture also enhances parasympathetic and anti-inflammatory effects [15]. However, there has always been a significant placebo effect observed in acupuncture users [16].

\section{Acupuncture applied to urologic conditions}

The beginnings of the use of acupuncture in urology are uncertain. For millennia, acupuncture in China was used to treat myriad symptoms. Certainly, these symptoms included urologic ones. However, published research directly investigating the efficacy of acupuncture applied to urology was lacking until recently. Traditionally urology was a procedure-based specialty, with urologists trained to achieve rapid resolution of disease either surgically or endoscopically. Urology has shifted in recent years towards clinic and office-based level of patient care; urologists are beginning to apply complementary and

Correspondence to: Wellman W Cheung, Department of Urology, State University of New York Downstate Medical School, 450 Clarkson Ave, Brooklyn, NY 11203, USA, E-mail: wellman.cheung@downstate.edu

Received: August 03, 2017; Accepted: August 18, 2017; Published: August 22, 2017 
alternative methods. Organizations such as the American Urological Association (AUA) and the Committee of Complementary and Alternative Medicine have recognized and advocated the integration of these alternative practices, including acupuncture [17].

As acupuncture has made a gradual entrance as an alternative therapy in urology, early studies were focused on pain control in renal colic and the spontaneous passage of stones. Acupuncture is known to dilate the ureter and has been proven more effective than conventional therapy for small stones [17]. There was also research that focused on overactive bladder and various irritative urinary symptoms, incontinence, infertility and impotence. This review article explores acupuncture as it is applied to renal colic and urolithiasis in pregnancy, overactive bladder, chronic pelvic pain syndrome and infertility. In general, evidence-based acupuncture is still in its primacy and results have been mixed. The paucity of studies is also compounded by the fact that many studies have been limited by study design, small sample size, and difficulty finding appropriate control groups. Nonetheless, existing findings point to some efficacy of acupuncture as a complement to conventional therapy.

\section{Renal/ureteral stone disease in pregnancy}

Renal stones are the most common cause of non-obstetrical abdominal pain causing hospitalization among pregnant patients [18]. They are associated with complications during pregnancy including premature labor, UTI and sepsis, and interference with progression of labor. Standard treatment of urolithiasis during pregnancy is similar to that outside of pregnancy. It ranges from conventional measures (i.e. bed rest, hydration, analgesics) to stent placement, ureteroscopy with stone manipulation, and percutaneous nephrostomy. Though more than $70 \%$ of stones pass with conservative treatment [19].

The first published study on the passage of ureteral stones by acupuncture was in 1976 by a group in Kweichow, China [20]. The study, published in 1976, was conducted in 1971-1972. The investigators reported a stone passage rate of $58 \%$ in a case study of 60 patients who received herbal medication and electro-acupuncture, a technique that involved apply electric energy to acupuncture needles. Furthermore, they observed that electro-acupuncture increased ureteral peristalsis in dogs but had no significant effect on urine flow in 11 humans.

The most regarded study linking acupuncture to the management of renal colic was a randomized control trial by Lin et al. [21] from Rizhao Shandong, China in 2005. Three hundred sixty patients with upper ureteral stones were randomized to electro-acupuncture, acupuncture and herbal medications. Stones were 4 to $6 \mathrm{~mm}$ by 6 to $18 \mathrm{~mm}$. Stone passage was confirmed by ultrasound or $\mathrm{x}$-ray. Complete stone passage or partial passage occurred in $91 \%$ from electro-acupuncture versus $80 \%$ and $76 \%$ for acupuncture and herbal medication, respectively, with complete stone passage in $72 \%$ versus $59 \%$ and $56 \%$, respectively. Although more effective, electro-acupuncture was more invasive because two electro-acupuncture needles were inserted to the parietal peritoneum under ultrasound guidance, superior and inferior to the stones. The authors theorized that electro-energy promoted ureteral peristalsis, promoted ureteral dilation that helped expulse the stones.

\section{Acupuncture in pregnancy}

Despite existing misconceptions, a recent systemic review found that acupuncture is not associated with any adverse effects during pregnancy when correctly applied [22]. Furthermore, an additional study performed on patients attempting to conceive via in-vitro fertilization (IVF), tested acupuncture applied to the Shenque (CV
8), Zhongji (CV 3), Guanyuan (CV 4), Qihai (CV 6), Zigong (EX-CA 1), Xuebai (SP 10) locations for 3 sessions in an observation group. Results showed a positive effect on estrogen level on based on hCG day, improved high-quality embryo rate, increased endometrial blood flow, and increased endometrial lining receptivity [23]. Traditional acupuncture has traditionally held that needling was "forbidden" at certain points during pregnancy. Yet, a recent study showed that 15 clinical trials ( $n=823$ women receiving 4549-7234 acupuncture treatments at one or more forbidden points) showed no increase in adverse outcomes like preterm birth (PTB) or still birth [24]. Other observational studies comparing women receiving acupuncture at forbidden points versus controls have shown no increase miscarriage, PTB, preterm prelabor rupture of membranes (PPROM), preterm labor (PTL), or threatened PTL in the forbidden points acupuncture groups. Taken together, these studies point to acupuncture as safe during pregnancy, with no known adverse effects.

\section{Chronic pelvic pain syndrome management}

Chronie pelvic pain syndrome (CPPS), of which chronic prostatitis (CP) is the male analog, is a major challenge to female healthcare providers, due to unclear etiology, multifactorial natural history, and management which often leads to unsatisfactory results. Direct medical costs approach $\$ 900$ million per annum [25]. Though the etiology is unclear, CPPS is often strongly associated with co-morbidities such as bladder or bowel dysfunction, sexual dysfunction, and other systemic or constitutional symptoms. Chronic lower urinary tract symptoms arising from CPPS and CP are intractable problems which lead to demonstrably lower quality of life. The etiology of chronic lower urinary tract symptoms is poorly understood, therefore, therapy has remained empiric. In acute exacberations, treatment is aimed at pain control which includes OTC drugs like paracetamol, ibuprofen, aspirin, or naproxen [26]. Tizanidine inhibits the CNS and has been shown to provide some pain relief in CPPS. Tricyclic antidepressants like amitriptyline (Elavil) and nortriptyline (Pamelor) are also sometimes used. Finally, selective serotonin reuptake inhibitors (SSRIs) are also sometimes prescribed and include fluoxetine (Prozac), paroxetine (Paxil), and sertraline (Zoloft).

A pilot study in China demonstrated acupuncture as an effective treatment of CP/CPPS symptoms, in conjunction with conventional therapy or alone [27]. In a controlled randomized clinical trial from 1994 Luo et al. [28], acupuncture was found to be superior to oral sulfamethoxazole in relieving symptoms and improving sexual function.

A recent meta-analysis combining 7 randomized control trial studies and 471 participants, compiled by Qin et al. [29], demonstrated efficacy of needle acupuncture in providing relief to pain in CP and CPPS. Pain management approaches incorporating acupuncture, sham acupuncture, and medicine (Levofloxacinand, Ibuprofen, and Tamsulosin) were compared in the meta-analysis. Results showed clear superiority of needle acupuncture over sham acupuncture in reducing NIH-Chronic Prostatitis Symptom Index, measurement of severity of CP/CPPS symptoms, and in improving responses in quality of life (QOL) surveys. Acupuncture showed similar symptom reduction as medicine, with no statistically significant difference in decreases in NIH-CPSI. A separately conducted meta-analysis by Liu and Wang in 2016, comprising patients from 10 randomized control studies, yielded similar results [30]. Acupuncture was clearly superior to sham acupuncture in improving NIH-CPSI, response rate, and quality of life. However, standard analgesic medication was superior to acupuncture in ameliorating urinary symptoms. It was furthermore observed that 
both standard medication and acupuncture significantly decreased IL$1 \mathrm{~B}$ in prostatic fluid analyzed for CP/CPPS.

Findings of individual randomized control studies support the meta-analyses. A 2008 Malaysian study by Lee compared acupuncture versus sham acupuncture in relieving pain from CP/CPPS [31]. $73 \%$ of patients from the acupuncture group versus $38 \%$ from the sham acupuncture group demonstrated a 6 point decrease in NIH-CPSI total score $(\mathrm{p}<0.02)$. Long term response rates at 24 weeks also showed treatment response rates of $32 \% v s .13 \%$ for the two groups $(\mathrm{p}<0.04)$. A Korean study by Chen and Nickel demonstrated even more impressive results [32]. 63participants with CP/CPPS were divided into 3 groups which received electro-acupuncture, sham acupuncture, and advice and exercise. After 6 weeks, the NIH-CPSI score for the EA patients decreased by an average of 6 points, versus 2 and 3 points for the other two groups respectively $(\mathrm{p}<0.01)$. Enzyme-linked immunosorbent assay was also used to measure levels of prostaglandins and betaendorphins in urine assays. EA patients had significantly decreased amounts compared to the other two groups ( $\mathrm{p}=0.023$ ). Thus, a clear predominance of evidence suggests therapeutic value for acupuncture in pain management of $\mathrm{CP} / \mathrm{CPPS}$.

\section{Overactive bladder}

Overactive bladder $(\mathrm{OAB})$ is characterized by urinary urgency, nocturia, daytime frequency and can also be associated with urge urinary incontinence. It causes impaired physical, social, and emotional functioning.The etiology of $\mathrm{OAB}$ is primarily neuromuscular, in which the detrusor muscle inappropriately contracts. The prevalence of $\mathrm{OAB}$ was found to be similar in women (16.9\%) and men (16\%) in the NOBLE study [33]. However, women were found to have greater frequency of severe symptoms in $\mathrm{OAB}$ such as incontinence. Guidelines for $\mathrm{OAB}$ treatment are provided by the American Urological Association (AUA) and the Society of Urodynamics, Female Pelvic Medicine and Urogenital Reconstruction (SUFU) [34]. First-line therapy includes behavioral therapy and education. Second-line therapy includes extended-release antimuscarinics such as transdermal oxybutynin. Beta-3 agonists can be used when other pharmacological treatments have failed. Third line therapy includes sacral neuromodulation or peripheral tibial nerve stimulation (PTNS). Intradetrusor injection of onabotulinumtoxinA may also be used in refractory cases.

Neuromodulation targets the stimulation of 33 either percutaneously and directly or distally at S3 dorsal root to reduce parasympathic outflow which inhibits bladder empting. In 1983, McQuire et al. [35] first reported the use of percutaneous nerve stimulation in 3 patients with detrusor instability. They placed stimulating electrodes at either the common peroneal or posterior tibial nerve. The placement of the electrodes originated from acupuncture points for inhibition of bladder activity. Then, in 1987, Stoller et al. [36] developed the Stoller Afferent Nerve Stimulator (SANS) and demonstrated its efficacy in monkeys. The Federal Drug Administration (FDA) later approved SANS for use in refractory overactive bladder in 2000 [37]. SANs paved the way for current percutaneous tibial nerve stimulation (PTNS) therapy, which is indicated in cases of overactive bladder refractory to conservative, pharmacological, and surgical management. PTNS involves placement a needle electrode at the posterior tibialnerve at the medial malleolus of the ankle. Stimulation of the needle electrode is transmitted to the sacral plexus which modulates bladder and pelvic floor muscles. The maximum tolerable treatment intensity is reached when the great toe curls. Induction is typically one 30-minute weekly treatment for 12 weeks, while maintenance frequency is determined by symptoms. The Interstim ${ }^{\star}$ Sacral Nerve Stimulation (Medtronic, Inc.), approved by the FDA in 1997, is a more invasive device that directly stimulates the dorsal nerve root of S3. Its use was approved by the FDA in 1997 [38].

A gynecological study in 2005 confirmed the efficacy of acupuncture in treating overactive bladder [39]. 85 women receiving either 4 weeks of acupuncture or sham/placebo acupuncture completed a 3-day voiding diary. Primary endpoint was the number of incontinent episodes over 3 days while secondary endpoints included frequency and urgency, voided volume, and bladder capacity. While there was no significant difference in the primary endpoint of the study, 59\% versus $40 \%$ of women in the acupuncture and placebo groups respectively experienced less incontinent episodes. Women in the acupuncture group had a statistically significant reduction in urinary frequency $(\mathrm{p}=0.013)$, proportion of voids with urgency $(\mathrm{p}=0.016)$, and voided volume $(\mathrm{p}=0.01)$. Another study by Yuan in 2015 compared the effectiveness of acupuncture tolterodine tartrate in reducing number of urinary urgency episodes, incontinence episodes, daytime frequency, nocturia episodes and increase in volume voided per micturition [40]. The trial demonstrated no statistical significant difference between the two groups, establishing equal efficacy of acupuncture and medical treatment in overactive bladder.

\section{Infertility}

Infertility is failure to conceive after 1 year of unprotected sexual intercourse, affecting $10-15 \%$ of couples. However, approximately $22 \%$ of infertility is due to male factor infertility. Recent studies have yielded results pointing to acupuncture as a potential therapeutic approach. The first published study on treatment of infertility by needle acupuncture was in 1984 by a German research group, inspired by its promising use in veterinary medicine. Fischl et al. [41] compiled a case study of 28 subfertile males. Each patient received a total of 10 acupuncture treatments over three weeks. A clear improvement was noted in sperm quality: total count, concentration and motility. In 1997, Sitterman et al. [42] conducted the first prospective controlled study comparing subfertile males in Israel over a 5-week period. Thirty-two males with low sperm quality were separated equally into two groups: treatment and placebo. The study reported statistically significant improvement in sperm viability and motility at one month after treatment. In another prospective controlled study of 40 males with idiopathic infertility, Pei et al. [43] reported an improvement in ultrastructural integrity of spermatozoa, by electron microscopy, after 5 weeks of acupuncture. Although these studies showed encouraging potential of acupuncture in improving semen quality, they were not ideally designed. Most findings were anecdotal and the studies lacked randomization.

The first published randomized controlled study appeared in 1998 when Xinyun et al. [44] randomized 108 males to receive acupuncture and clomiphene ( $25 \mathrm{mg} \mathrm{qD}$ ) versus clomiphene monotherapy. Subjects were diagnosed with idiopathic normo gonadotrophic oligospermia (20 million $/ \mathrm{mL})$. Pregnancy or normalization of semen parameters was achieved in $74 \%$ of patients receiving acupuncture and clomiphene combination therapy, compared with $52 \%$ of those receiving clomiphene monotherapy, a statistically significant difference. Nonetheless, a recent systemic review conducted by Jerng and Jo in the Asian Journal of Andrology aggregated findings from 14 databases on 4 RCTs on the efficacy of acupuncture on treating male infertility. However, there were more ambiguous findings [45]. The primary outcomes sought were sperm motility, sperm concentration, pregnancy rate, and adverse events. Findings showed that while acupuncture increased sperm concentration (CI 4.91-7.92) and percentage of sperm with rapid progression (4.38-8.32), there was no statistically significant difference 
in pregnancy rate (CI 0.70-3.69). Zero patients experienced adverse effects from acupuncture treatment. However, the lack of difference in pregnancy rate may have been due to insignificant power and lack of patients available for analysis in the systemic review [45]. Clearly, greater numbers of RCTs are needed to elucidate the true relationship between acupuncture and efficacy in treating male infertility.

\section{Conclusion}

Since its reintroduction to Western medicine in the 1970s, acupuncture has made a graduala comeback into evidence-based medicine. Once plagued by the stigma of pseudoscience and a lack of any scientifically proven mechanism of action, both these issues have gradually resolved themselves as Western practitioners have found greater liberalism and willingness to incorporate traditional and alternative medicine into mainstream practice.

In recent years, an increasing number of randomized control studies and meta-analyses have demonstrated the efficacy of acupuncture in various urologic conditions. Urolithiasis is the most common cause of abdominal pain in pregnancy, and case studies and there is a demonstrated efficacy of acupuncture in increasing frequency of passage of urinary stones, particularly those less than $5 \mathrm{~mm}$. Perhaps more significantly, a myriad of randomized controlled trials has demonstrated acupuncture as having clear and demonstrable effects in pain management of nephrolithiasis and shock wave lithotripsy patients. Patients undergoing acupuncture had decreased pain scores. Those undergoing acupuncture as an adjunct to conventional medical anesthesia via patient administered pump used less analgesic than controls. Acupuncture proved equally effective in pain management of chronic prostatitis and chronic pelvic pain syndrome. Randomized controlled trials and meta-analyses have showed repeated and statistically significant decreases in NIH-CPSI score in patients undergoing acupuncture versus sham acupuncture treatments. Analysis of prostaglandins and beta-endorphins in urine were also significantly increased in acupuncture patients versus controls. Electro-acupuncture also demonstrated efficacy in treatment of overactive bladder. Primary endpoints such as incontinent episodes, urgency, frequency, and post-void urinary volume were all ameliorated in randomized controlled trials over the past couple decades. Furthermore, FDA-approved devices used to treat overactive bladder such as the SANS and Interstim ${ }^{\bullet}$ Sacral Nerve Stimulation Device by Medtronic drew direct inspiration from electro-acupuncture approaches validated by these studies. Finally, male infertility is an area which acupuncture demonstrated therapeutic efficacy. Patients undergoing acupuncture displayed statistically significant increases in primary and secondary endpoints such as sperm count, motility, concentration, and even improvements in spermatozoa structure on electron microscopy. Though studies have yet to directly associate acupuncture with increased pregnancy rates, this is one area for future randomized controlled trials.

As acupuncture continues to gain ground as a viable adjunct treatment to conventional evidence-based medicine, it is likely further RCTs, systemic reviews, and meta-analyses will be conducted, elucidating the efficacy of acupuncture on a host of age-old urologic pathologies. As the findings of these studies emerge, urologists and pain management practitioners will be better able to make informed recommendations on the therapeutic efficacy and potential adverse effects of this ancient treatment.

\section{References}

1. White A, Ernst E (2004) A brief history of acupuncture. Rheumatology (Oxford) 43 : 662-663. [Crossref]

2. Cheng X (1987) Chinese Acupuncture and Moxibustion (1st ed.). Foreign Languages Press.

3. Lu GD, Needham J (2002) Celestial Lancets: A History and Rationale of Acupuncture and Moxa.

4. Joseph H (1995) Acupuncture energetics: a clinical approach for physicians. Medical Acupunture Publishers. p.9.

5. Reston, James (1971) Now, about my operation in Peking; Now, let me tell you about my appendectomy in Peking. New York Times. pp. 1-2.

6. Fan AY (2012) The first acupuncture center in the United States: an interview with Dr Yao Wu Lee, Washington Acupuncture Center. J Integr Med.

7. Food and Drug Administration, Center for Devices and Radiological Health (1996) Acupuncture Needle Status Change" letter, www.fda.gov/cdrh.

8. Brian F, Richard E (2004) Council of Acupuncture and Oriental Medicine Associations and Foundation for Acupuncture Research. Acupuncture and electroacupuncture: evidence-based treatment guidelines.

9. World Health Organization (2002) Acupuncture: Review and analysis of reports on controlled clinical trials. WHO Geneva.

10. American Society of Anesthesiologists Task Force on Chronic Pain Management American Society of Regional Anesthesia and Pain Medicine (2010). "Practice guidelines for chronic pain management: an updated report by the American Society of Anesthesiologists Task Force on Chronic Pain Management and the American Society of Regional Anesthesia and Pain Medicine." Anesthesiology 112: 810-833. [Crossref]

11. Han JS (2000) Opioid and antiopioid peptides: A model of Yin-Yang balance in acupuncture mechanism of pain modulation. In Clinical Acupuncture: Scientific Basis. edited by G. Stux and R. Hammerschlag, Berlin: Springer-Verlag. p51-68.

12. "Hard to swallow". Nature. 448 (7150): 105-106. 2007.Ulett, GA (2002) "Acupuncture". In Shermer, M. The Skeptic: Encyclopedia of Pseudoscience. ABCCLIO. p. 283291.

13. Berman BM, Langevin HM, Witt CM, Dubner R (2010) Acupuncture for chronic low back pain. $N$ Engl J Med 363: 454-461. [Crossref]

14. Wu MT, Hsieh JC, Xiong J, Yang CF, Pan HB, et al. (1999) Central nervous pathway for acupuncture stimulation: localization of processing with functional MR imaging of the brain--preliminary experience. Radiology 212: 133-141. [Crossref]

15. Madsen MV, Gøtzsche PC, Hróbjartsson A (2009) Acupuncture treatment for pain systematic review of randomised clinical trials with acupuncture, placebo acupuncture, and no acupuncture groups. $B M J$ 338: a3115. [Crossref]

16. Hirsch IH (2000) Integrative urology: a spectrum of complementary and alternative therapy. Urology 56: 185-189. [Crossref]

17. Zhang WR (1992) Clinical observation of acupuncture in treating kidney and ureter stones. Chinese Acupuncture and Moxibustion. 12: 5-6.

18. Folger GK (1955) Pain and pregnancy; treatment of painful states complicating pregnancy, with particular emphasis on urinary calculi. Obstet Gynecol 5: 513-518. [Crossref]

19. Parulkar BG, Hopkins TB, Wollin MR, Howard PJ Jr, Lal A (1998) Renal colic during pregnancy: a case for conservative treatment. J Urol 159: 365-368. [Crossref]

20. Acute Abdominal Condition Research Group in Kweichow, China (1976) Clinical and experimental studies on "general attack therapy" of ureteral stones. Chin Med J (English) 2: 25-32.

21. Lin Q1, Li X, Han J, Leng J (2005) Electro-acupuncture treatment for the upper segment ureterolithiasis under B-ultrasonography, J Tradit Chin Med 25: 13-15. [Crossref]

22. Park J, Sohn Y, White AR, Lee H (2014) The safety of acupuncture during pregnancy: a systematic review. Acupunct Med 32: 257-266. [Crossref]

23. Chen Q, Hau C (2015) [Impacts on pregnancy outcome treated with acupuncture and moxibustion in IVF-ET patients]. Zhongguo Zhen Jiu 35: 313-317. [Crossref]

24. Carr DJ (2015) The safety of obstetric acupuncture: forbidden points revisited Acupunct Med 33: 413-419. [Crossref]

25. Mathias SD, Kuppermann M, Liberman RF, Lipschutz RC, Steege JF (1996) Chronic pelvic pain: prevalence, health-related quality of life, and economic correlates. Obstet Gynecol 87: 321-327. [Crossref] 
26. American College of Obstetricians and Gynecologists (ACOG) (2004) Chronic Pelvic Pain. Washington (DC): ACOG (ACOG practice bulletin; no. 51).

27. Jillian C (2007) A pilot study on acupuncture for lower urinary tract symptoms related to chronic prostatitis/chronic pelvic pain. Chin Med 2:1.

28. Luo YN (1994) Clinical research on treatment of chronic prostatitis with acupuncture. World Journal of Acupuncture-Moxibustion 4: 7-14.

29. Qin Z, Wu J, Zhou J, Liu Z (2016) Systematic Review of Acupuncture for Chronic Prostatitis/Chronic Pelvic Pain Syndrome. Medicine (Baltimore). 95: e3095. [Crossref]

30. Liu BP, Wang YT, Chen SD (2016) Effect of acupuncture on clinical symptoms and laboratory indicators for chronic prostatitis/chronic pelvic pain syndrome: a systematic review and meta-analysis. Int Urol Nephrol 48: 1977-1991. [Crossref]

31. Lee SW, Liong ML, Yuen KH, Leong WS, Chee C, et al. (2008) Acupuncture versus sham acupuncture for chronic prostatitis/chronic pelvic pain. Am J Med 121: e1-7. [Crossref]

32. Lee SH, Lee BC (2009) Electroacupuncture relieves pain in men with chronic prostatitis/chronic pelvic pain syndrome: three-arm randomized trial. Urology 73 : 1036-1041. [Crossref]

33. Stewart WF, Van Rooyen JB, Cundiff GW, Abrams P, Herzog AR, et al. (2003) Prevalence and burden of overactive bladder in the United States. World J Urol 20: 327-336. [Crossref]

34. Brotn T (2012) Overactive bladder guidelines released. Medscape Medical News.

35. McGuire EJ, Zhang SC, Horwinski ER, Lytton B (1983) Treatment of motor and sensory detrusor instability by electrical stimulation. J Urol 129: 78-79. [Crossref]

36. Stoller M, Copeland S, Millard RJ, Murnaghann GF (1987) The Efficacy of Acupuncture in Reversing the Unstable Bladder in Pig-tailed Monkeys. J Urol 137: 104A.

37. Federal Drug Administration (2000) Premarket approval of non-implanted, peripheral nerve stimulator for pelvic floor dysfunction. Fed Regist 65.

38. [No authors listed] (1998) Medtronic, Inc.; premarket approval of the Interstim Sacral Nerve Stimulation (SNS) System--FDA. Notice. Fed Regist 63: 4457. [Crossref]

39. Emmons SL, Otto L (2005) Acupuncture for overactive bladder: a randomized controlled trial. Obstet Gynecol 106: 138-143. [Crossref]

40. Yuan Z, He C, Yan S, Huang D, Wang H, et al. (2015) Acupuncture for overactive bladder in female adult: a randomized controlled trial. World J Urol 33: 1303-1308. [Crossref]

41. Fischl F, Riegler R, Bieglmayer C, Nasr F, Neumark J (1984) [Modification of semen quality by acupuncture in subfertile males]. Geburtshilfe Frauenheilkd 44: 510-512. [Crossref]
42. Siterman S, Eltes F, Wolfson V, Zabludovsky N, Bartoov B (1997) Effect of acupuncture on sperm parameters of males suffering from subfertility related to low sperm quality. Arch Androl 39: 155-161. [Crossref]

43. Pei J, Strehler E, Noss U, Abt M, Piomboni P, et al. (2005) Quantitative evaluation of spermatozoa ultrastructure after acupuncture treatment for idiopathic male infertility. Fertil Steril 84: 141-147. [Crossref]

44. Xinyun H (1998) Acupuncture plus medication for male idiopathic oligospermaticsterility. Shanghai J Acupunc tMoxibustion. 2:35-37.

45. UiMin Jerng, Jun-Young Jo, Seunghoon Lee, Jin-Moo Lee, Ohmin Kwon (2014) The effectiveness and safety of acupuncture for poor semen quality in infertile males: a systematic review and meta-analysis. Asian J Androl 16: 884-891. [Crossref]

46. Barnes LL (2005) Needles, Herbs, Gods, and Ghosts: China, Healing, and the West to 1848. Harvard University Press.

47. Jackson M (2011) The Oxford Handbook of the History of Medicine. Oxford Handbooks in History. OUP Oxford. p. 610.

48. Colquhoun D, Novella SP (2013) Acupuncture is theatrical placebo. Anesth Analg 116 1360-1363. [Crossref]

49. Yang DL (1989) Acupuncture treatment of 182 cases of abdominal colic due to calculi in the urinary system. $J$ Tradit Chin Med 9: 247-248. [Crossref]

50. Chen L (1991) 408 cases of urinary calculus treated by auriculoacupoint pressure. $J$ Tradit Chin Med 11: 193-195. [Crossref]

51. Wang SM, Kain ZN (2001) Auricular acupuncture: a potential treatment for anxiety Anesth Analg 92: 548-553. [Crossref]

52. Resim S, Gumusalan Y, Ekerbicer HC, Sahin MA, Sahinkanat T (2005) Effectiveness of electro-acupuncture compared to sedo-analgesics in relieving pain during shockwave lithotripsy. Urol Res. 33: 285-290. [Crossref]

53. Quatan N, Bailey C, Larking A, Boyd PJ, Watkin N (2003) Sticks and stones: use of acupuncture in extracorporeal shockwave lithotripsy. $J$ Endourol 17: 867-870. [Crossref]

54. Li L, Jiang S, Zhong Y (1994) The application of acupuncture anesthesia in cystoscopy. $J$ Tradit Chin Med 14: 30-31. [Crossref]

55. Yaman LS, Kiliç S, Sarica K, Bayar M, Saygin B (1994) The place of acupuncture in the management of psychogenic impotence. Eur Urol 26: 52-55. [Crossref]

56. Kho HG, Sweep CG, Chen X, Rabsztyn PR, Meuleman EJ (1999) The use of acupuncture in the treatment of erectile dysfunction. Int J Impot Res 11: 41-46. [Crossref]

57. Cui X, Li X, Peng W, Zhou J, Yu J, et al. (2015) Acupuncture for erectile dysfunction: a systematic review protocol. BMJ Open 5: e007040. [Crossref]

Copyright: (C2017 Esdaille A. This is an open-access article distributed under the terms of the Creative Commons Attribution License, which permits unrestricted use, distribution, and reproduction in any medium, provided the original author and source are credited. 